BREAST CANCER

\title{
HER2-targeted agents overcome resistance
}

The majority of women with HER2-positive breast cancer will respond to trastuzumab and/or other HER2-targeted therapies. Nonetheless, most will ultimately develop resistance to first-line and later-line therapies, thus necessitating new agents that can overcome acquired resistance. Now, new data presented at the 2019 San Antonio Breast Cancer Symposium demonstrate the efficacy of two new treatment options for patients with heavily pretreated metastatic HER2-positive breast cancer.

In the phase III HER2CLIMB trial, a total of 612 patients with metastatic HER2-positive breast cancer who had received a median of four prior lines of therapy, including trastuzumab, pertuzumab and trastuzumab emtansine (TDM-1),

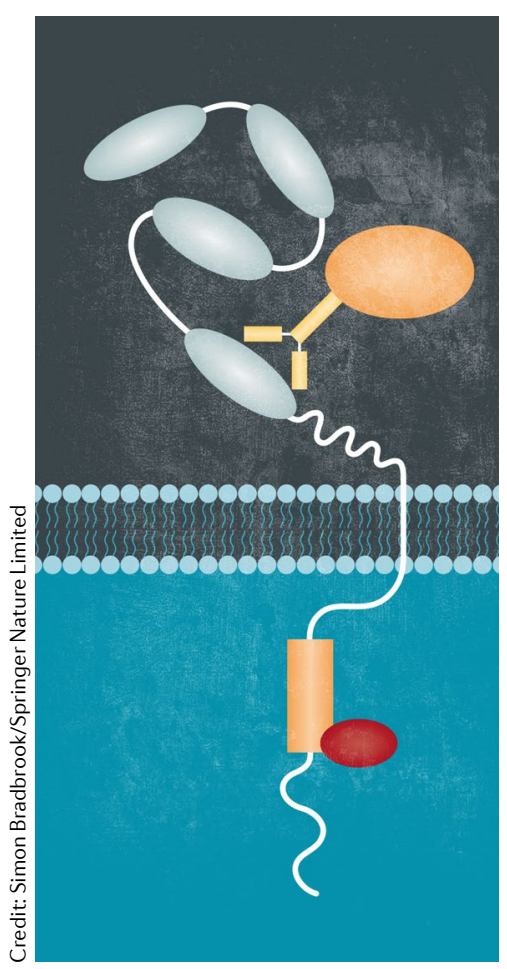

were randomized (2:1) to receive either tucatinib, a HER2-tyrosinekinase inhibitor that binds to the internal domain of HER2, or placebo in combination with trastuzumab plus capecitabine. The primary end point was progression-free survival (PFS) in a prespecified population of 480 patients.

Median PFS was 7.8 months in the tucatinib group versus 5.6 months in the placebo group (HR 0.54, 95\% CI $0.42-0.71 ; P<0.001)$. This increase was reflected in improved overall survival (OS) in patients receiving tucatinib (21.9 months versus 17.4 months, respectively: HR 0.66 , 95\% CI 0.50-0.88; $P=0.005)$, which was obtained from analysis of the total population. Grade $\geq 3$ adverse events were more common in the tucatinib group (in $55 \%$ versus $48.7 \%$ of patients) and included hand-foot syndrome (in 13.1\%) and diarrhoea (in $12.9 \%$ ).

In a subgroup analysis, patients with brain metastases at baseline derived greater levels of benefit from tucatinib, most likely reflecting the greater blood-brain barrier (BBB) permeability of this agent. Nonetheless, benefit from tucatinib was not restricted entirely to this population, implying that combined inhibition of the intracellular and extracellular domains of HER2 is effective, even in heavily pretreated patients.

In another study presented at the Symposium, patients with heavily pretreated metastatic HER2-positive breast cancer (who received a median of 6 prior therapies, including trastuzumab and T-DM1 in all patients) received the antibody-drug conjugate trastuzumab deruxtecan in a single-arm, two-part, phase II open-label study. Owing to high levels of toxicity with higher doses, a $5.4 \mathrm{mg} / \mathrm{kg}$ dose was selected for the efficacy phase of the trial.

A total of 184 patients received trastuzumab deruxtecan, of whom $60.9 \%$ had an objective response, with a median PFS duration of 16.4 months. Disease control was achieved in virtually all patients (97.3\%). Median OS was not reached at the time of data analysis cutoff (estimated 1-year OS 86.2\%). Grade $\geq 3$ adverse events occurred in $48.4 \%$ of patients, including neutropenia (in 19.6\%) and anaemia (in $8.2 \%$ ).

These observations, from a population of patients with TDM-1resistant HER2-positive breast cancer, suggest that resistance to the cytotoxic payload, as well as alterations in HER2, can both have a role in acquired resistance. However, the investigators also note that the increased drug:antibody ratio, higher dose administered, and/or greater cellular permeability of the cytotoxic payload of trastuzumab deruxtecan, relative to T-DM1, might all explain the activity of this agent in T-DM1 resistant disease.

In summary, the promising outcomes of these two trials provide new treatment approaches for patients with heavily pretreated metastatic HER2-positive breast cancer. Both studies also provided evidence of greater efficacy in patients with brain metastases, in subgroup analyses. These observations merit further testing of these agents in patients with brain-metastatic HER2-positive cancers, owing to the limited number of effective BBB-permeable targeted therapies currently available.

Peter Sidaway

ORIGINAL ARTICLES Murthy, R. K. et al. Tucatinib, trastuzumab, and capecitabine for HER2 positive metastatic breast cancer. N. Engl.J. Med. https://doi.org/10.1056/NEJMoa1914609 (2019)| Modi, S. et al. Trastuzumab deruxtecan in previously treated HER2-positive breast cancer. N. Engl.J. Med. https://doi.org/10.1056/ NEJMoa1914510 (2019) 